\title{
Endocrine and metabolic diseases
}

\section{Treatment of thyroid disease: I}

\author{
D C EVERED
}

British Medical fournal, 1976, 1, 264-266

\section{Hyperthyroidism and Graves's disease}

Hyperthyroidism is a potentially serious condition and therefore merits treatment. Three specific methods of treatment are available: antithyroid drugs, partial thyroidectomy, and radioactive iodine.

\section{ANTITHYROID DRUGS}

It is possible to interfere pharmacologically with each stage of thyroid hormone synthesis, release, and transport. The usual drugs, however, are either inhibitors of organification (thionamides) or less frequently inhibitors of iodide transport (potassium perchlorate). Iodides, lithium compounds, and betaadrenergic blockers have also been used, although the last group has no direct action upon the thyroid.

Thionamides-The major drug in this group is carbimazole, which interferes with organification. Propylthiouracil may also inhibit the peripheral monodeiodination of thyroxine, but there is no good evidence that this action contributes to its therapeutic effect in man. Hyperthyroidism may be controlled in all patients with adequate doses of thionamides, but these drugs have a short half life and must, therefore, be administered in divided doses. The initial dose of carbimazole is $10-15 \mathrm{mg}$ every six hours. A biochemical response is seen within one to two weeks, although the clinical response takes longer. The dose may be reduced and adjusted on the basis of clinical and biochemical observations as the hyperthyroidism comes under control. The

Royal Victoria Infirmary, Newcastle upon Tyne NE1 4L.P D C EVERED, MD, MRCP, consultant physician

alternative course is to maintain a full blocking dose of carbimazole and add $0.15 \mathrm{mg}$ thyroxine to the patient's medication when the hyperthyroidism is under control. This latter procedure has the advantage that fluctuations in the patient's clinical state are avoided as the dose is progressively adjusted. Hypersensitivity reactions and agranulocytosis may occur but are rare with carbimazole. Propylthiouracil or methylthiouracil may be used as alternatives if hypersensitivity to carbimazole occurs. The initial dose of these drugs is $300-400 \mathrm{mg}$ daily.

Potassium perchlorate-This agent interferes with iodide transport and should only be used in patients who have developed hypersensitivity to the thionamides, since there is a higher risk of blood dyscrasias with this drug. The initial dose is $200 \mathrm{mg}$ every six hours. Patients receiving perchlorate should not be given iodide since too much iodide will overcome the inhibition of transport.

Iodides-The actions of iodides on the thyroid are complex. Their use in the preparation of the patient for thyroidectomy has now been superseded by the combined use of carbimazole and thyroxine. The sole remaining use for iodides is in the management of thyroid crisis. The most suitable preparation is potassium iodide tablets $(60 \mathrm{mg})$, although the traditional Lugol's iodine may be used.

Lithium compounds-Lithium compounds reduce the synthesis of thyroid hormone and also block hormone release. They have major metabolic and endocrine effects unrelated to the thyroid in addition to their other toxic effects. There is no justification for their use since safer and more specific drug treatments are available.

Beta-adrenergic blockers-Several clinical features of hyperthyroidism may be attributed to increased adrenergic activity. These effects will be improved by beta-blocking agents. There is no justification for using these drugs routinely since specific agents which control all the features of hyperthyroidism are available. The major use of beta-blockers, as an adjunct to other measures, is in the management of arrhythmias or in impending 
or actual thyroid crisis to minimise the cardiovascular effects of preformed hormone. The only beta-blocker which should be used for this purpose is propranolol since other preparations have intrinsic sympathomimetic activity.

\section{PARTIAL THYROIDECTOMY}

Partial thyroidectomy generally provides the most certain method of eliminating hyperthyroidism and goitre. There is also growing evidence that this procedure has a major effect on the immunological disturbances of Graves's disease. Surgery should never be performed in the unprepared patient. Preparation should be carried out with carbimazole to render the patient euthyroid and with thyroxine to eliminate the risk of thyroidstimulating hormone stimulation of the blocked gland as described above.

Surgery should reduce the thyroid mass sufficiently to cure the hyperthyroidism but leave sufficient secretory tissue for the patient's needs. This is not always achieved. The incidence of hypothyroidism has been reported to lie at $3-35 \%$ and recurrent hyperthyroidism at $1 \cdot 2-28 \%$. Many factors play a part in these wide variations, including remnant size, the degree of lymphocytic infiltration of the gland, the extent of thyroid regeneration, the alteration in immunological status, and the precision of the diagnostic techniques used to identify abnormalities of thyroid function. The other main complications of thyroid surgery are hypoparathyroidism, recurrent laryngeal palsy, and thyroid crisis.

\section{RADIOIODINE TREATMENT}

Radioiodine treatment was first introduced in 1942. It is almost invariably carried out using ${ }^{131} \mathrm{I}$ (early studies suggest that ${ }^{125}$ I treatment has no appreciable advantage). This treatment is convenient for the patient, and early fears that there might be an increased frequency of thyroid carcinoma, leukaemia, or congenital abnormalities in the children of patients have been proved groundless. Radioiodine is generally reserved for patients over 40 and should never be given during pregnancy.

The administration of radioiodine may be followed by a transient increase in thyroid size, and a release of preformed hormone into the circulation. Neither of these events are usually associated with clinical problems, although the latter may exacerbate an arrhythmia in a severely hyperthyroid patient. The major disadvantages, however, are that there is generally a delay in controlling the hyperthyroidism and a high incidence of hypothyroidism. The delay before clinical improvement occurs is variable and cannot be predicted. It is generally optimistic to expect biochemical and clinical control in less than two to three months and this period may be much longer. The treatment dose of radioiodine should, therefore, be followed by a period on antithyroid drugs to achieve early clinical control. Some patients will require a second dose of radioiodine and a minimum period of one year should be allowed between doses.

The most important and frequent complication of radioiodine treatment is hypothyroidism, and $10-15 \%$ of patients develop this during the first year after treatment and a further $3-6 \%$ became hypothyroid annually thereafter. The prevalence of hypothyroidism after 10 years lies at $40-60 \%$ and approaches $80 \%$ after 15 years. Many factors modify the biological effects of radioiodine and only some of these can be measured; thus it is not possible to calculate the "correct" dose for a patient.

Radioiodine is not an ideal treatment for hyperthyroidism but is, nevertheless, the some satisfactory form of destructive treatment for some patients.

\section{MANAGEMENT OF THE INDIVIDUAL PATIENT}

Neonatal hyperthyroidism-This is an acute self-limiting condition which will remit in three to four months. Treatment should be with iodides and antithyroid drugs for this period and the dose should be adjusted on clinical grounds.

Hyperthyroidism in children-This generally occurs in the second decade. Antithyroid drug treatment should be used for one to three years, and if subsequent relapse occurs the patient should be treated surgically.

Hyperthyroidism in young adults-There are two recognised courses of action open to this age group. Firstly, the patient may be treated with antithyroid drugs (and thyroxine) for one to two years. The relapse rate is about $30 \%$ after one year and $45-55 \%$ after 10 years. Relapse should be treated surgically. The practice of giving patients repeated courses of antithyroid drugs is to be deprecated. Alternatively partial thyroidectomy may be performed as soon as the patient has been adequately controlled with antithyroid drugs and thyroxine. The advantage is that the hyperthyroidism is treated directly, the immunological status of the patient is altered, and the risk of severe ophthalmic problems is reduced. The disadvantage is that surgery is performed on about half the patients who will achieve long-term remission after a single course of antithyroid drugs. Patients in this age group, therefore, should be treated with antithyroid drugs and surgery should be reserved for a relapse. Surgery should be considered in the first episode in patients with severe disease, a large goitre, or an ophthalmopathy of any severity.

Hyperthyroidism in pregnancy-This should usually be controlled with antithyroid drugs together with thyroxine to prevent fetal hypothyroidism. Breast-feeding must be avoided if it is necessary to continue treatment during the puerperium since the thionamide drugs are excreted in the milk.

Hyperthyroidism in middle-aged and elderly patients-Patients in this group should normally be treated with radioiodine followed by antithyroid drugs after a week. Thyroxine should be added when the patient becomes euthyroid. Drugs should be discontinued after 12 months to reassess the patient's thyroid state and a further dose of radioiodine given if the patient remains hyperthyroid. Hyperthyroidism should be controlled with drugs before administering radioiodine in patients with major cardiovascular problems. It is, of course, necessary to discontinue the antithyroid drugs for one week before and one week after the dose. Subsequent control should follow the lines indicated above.

Nodular goitre-Hyperthyroidism due to a nodular goitre should normally be treated surgically (after drug control) in the younger patient, but may be treated by radioiodine in the older patient if pressure symptoms are not present.

Thyroid crisis-The management of crisis should include intravenous sodium iodide, oral carbimazole, propranolol, and parenteral steroids. Supportive treatment must provide intravenous fluids, salicylates to counteract fever, oxygen administration to combat anoxia, and digoxin and diuretics if cardiac failure occurs.

\section{EXTRATHYROIDAL MANIFESTATIONS OF GRAVES'S DISEASE}

Ophthalmopathy-Minor degrees of ophthalmopathy do not require treatment. Ophthalmic problems are less troublesome if the patient is rendered permanently euthyroid early in the disease. (1) Discomfort and grittiness in the eyes may generally be relieved by methyl cellulose eye drops. (2) Lid retraction may be distressing to patients and may be relieved in some by guanethidine eye drops. These do, however, have the disadvantage that they cause conjunctival irritation in many patients. (3) Proptosis and congestive ophthalmology: these should normally be managed by a specialist unit. Steroids and other immunosuppressives, tarsorrhaphy, orbital decompression, and orbital irradiation may all play a part in the management of these problems.

Dermopathy-Minor degrees of localised myxoedema do not require treatment. More extensive lesions are best treated with local steroid preparations under occlusive polythene dressings. Areas which are resistant to this form of treatment will usually respond to local infiltration with triamcinolone. 


\section{Hypothyroidism}

There is general agreement that all patients with symptomatic hypothyroidism should receive replacement treatment.

\section{MANAGEMENT}

Thyroxine should be used in the routine management of hypothyroidism. The initial dose should not exceed $0.05 \mathrm{mg}$ daily in patients over 40 ; an initial dose of $0.1 \mathrm{mg}$ daily may be used in younger patients. The dose should be increased by increments until the patient is clinically and biochemically euthyroid. The optimum dose varies between 0.1 and $0.2 \mathrm{mg}$ daily and higher doses are very rarely required. There is no need for triiodothyronine or tablets combining thyroxine and triiodothyronine in routine management, since these confer no additional symptomatic benefit, side effects are more common, and serum triiodothyronine levels are normal on thyroxine as there is a substantial extrathyroidal conversion of thyroxine to triiodothyronine. A raised serum thyroxine concentration is not, therefore, an essential prerequisite for adequate replacement treatment as has been claimed. There is only one particular indication for the long-term maintenance of a patient on triiodothyronine in preference to thyroxine: in patients who have had total thyroid ablation for thyroid carcinoma treatment should be stopped at regular intervals so that scanning procedures can be carried out. The replacement treatment can be discontinued for a considerably shorter time if triiodothyronine is used. It is important to ensure that adequate hormone is given to suppress thyroid-stimulating hormone secretion in these patients since this is a major part of their management.

Replacement treatment with thyroxine must be introduced cautiously in patients with ischaemic heart disease, in whom it may not be possible to give full replacement doses. The addition of a beta-adrenergic blocker such as propranolol, which can be increased incrementally with the thyroxine, may allow patients with angina pectoris to tolerate a larger dose of thyroxine than would otherwise be possible.

There is no general agreement on the best treatment for hypothyroid (myxoedema) coma. It is common practice to give thyroxine in doses of $0.05 \mathrm{mg}$ daily by mouth combined with triiodothyronine $20-40 \mu \mathrm{g}$ daily by intramuscular injection together with hydrocortisone hemisuccinate $100 \mathrm{mg}$ twice daily. Supportive treatment with fluids, assisted respiration, and treatment for cardiac failure or arrhythmias should be provided. The body temperature should be slowly raised to normal. It must be remembered that over-enthusiastic therapeutic activity can be as dangerous as inactivity in patients with coma.

The monthly MIMS index of proprietary preparations available in Britain includes the following trade names for the main drugs mentioned in this article (other than brood groups such as corticosteroids). Carbimazole: Neo-mercazole; potassium perchlorate: Peroidin; thy roxine: Eltroxin.

\title{
Problems of Childhood
}

\section{Consultation, diagnosis, and management}

\author{
ROY MEADOW
}

British Medical fournal, 1976, 1, 266-267

The articles on problems of childhood which will be published during the next few months deal with common problems and worries that confront families and the doctors who help them. The articles have a strong emphasis on important aspects of management for the doctor responsible for primary care. Throughout the articles it is implicit that the doctor dealing with the case will be following the rules of a paediatric consultation. There are certain rules to be obeyed and guidelines to be followed in dealing with children. The rules will be adapted according to ones own personality, experience, and philosophy as a doctor, and also according to the situation, the child, and the family. Moreover, like all the best rules, the rules applicable to the paediatric consultation will need to be broken occasionally.

\section{Consultation}

If the child is very ill he is likely to be in bed at home with a parent nearby, but most children will be seen in a surgery

\footnotetext{
Department of Paediatrics and Child Health, University of Leeds, Leeds LS1 3ET

ROY MEADOW, FRCP, DCH, senior lecturer and consultant paediatrician
}

or a clinic. In that setting, while the infant can easily be at ease and happy in his mother's arms, to make the toddler or school child at ease requires tact, care, and planning.

Tiny chairs may be comfortable, but the doctor is much more likely to communicate well with the child if he is on the same level rather than towering over the child. Communication is also easier if the chairs are at right angles to the desk, and the child within touching distance: this avoids any semblance of the headmaster/pupil relationship, with the master on a big chair confronting across a desk a pupil in a smaller chair. A few models and blocks $(2.5 \mathrm{~cm}$ coloured wooden cubes are best) on the desk give children of most ages something to watch or play with. A more generous selection of toys in the corner of the room will occupy accompanying brothers or sisters.

We all like to be called by our names and children are no exception. It makes them feel more secure to be greeted with "Hello Kevin" than "Hello little chap." (I sometimes wish that the thousands of elderly patients who get addressed as "Gran" and the children who are labelled "Little chap" or "Titch" returned the compliment by calling their doctor "Doc" or "Dad.")

The main practical problem of the paediatric consultation is the time it may take. Unless adequate time can be spent on children with recurrent and chronic disorders there is little chance of effective help. It is surely better to spend a lot of time trying to get to the bottom of a problem and to help with it than to spend the same amount of time over a succession of repeated brief visits and get nowhere. 